water content and blood flow in the brain. Furthermore the forth factor which is thought of the summation of the above three factors; cerebral architectural changes particularly enlargement and reduction of the cerebral blood vessel bed, have the most close relation to the change of attenuation of ultrasound. Therefore authors are going to search the relation between attenuation of ultrasound and cerebral architectural changes.

\title{
m-3. Biochemical Changes in Cerebral Edema
}

\author{
Shozo Nakazawa, Yuichi Komai, Keishi Kobayashi, Yoshifumi Furusawa, \\ Kenichi Tanimura, Noboru MiYasawa and Komei UekI \\ Department of Neurosurgery, Brain Research Institute, Niigata University
}

Cerebral edema was produced in rats and cats by freeze treatment or by implanted balloons and its metabolic activity was studied. There observed an increase in water and sodium and a decrease in potassium content of the white matter that resulted in a sharp of $\mathrm{Na} / \mathrm{K}$ ratio.

No significant difference in the content level of total protein, total lipid, RNA and DNA was shown between control and experimental. On the other hand there observed a sharp decrease of the level of creatine phosphate and a mild decrease in ATP level.

Radioactive amino acid incorporation into protein increased markedly after the production of edema and $\left[5-{ }^{3} \mathrm{H}\right]$ uridine incorporation into RNA also increased.

An increase in the level of proteolipid protein content of gray matter and a decrease in that of white matter were observed. On the other hand $\left[2-{ }^{14} \mathrm{C}\right]$ glycine incorporation into proteolipid protein increased markedly both in gray and white matter.

Respiratory response to cationic and electrical stimulation decreased. Na-K ATPase, GDC, SDH, MAO activities were not affected.

\section{m-4. Ultrasonic Attenuation in Cerebral Compression and Acute Brain Swelling}

\author{
Susumu Ishikawa, Hiromichi Hibino, Junji Goishi, Satoru Ohtani, \\ Shigeru Watanabe, Chiaki Inoguchl and Yasuo TAKeUChI \\ 2nd Department of Surgery, Hiroshima University School of Medicine
}

In the previous report, basing on the experiments of brain compression produced by expansion of an epidural balloon, we defined 5 successive stages of 
cerebral compression indicated by changes in respiration, pulse, and systemic blood pressure. Also, we presented experimental evidence that rapid evacuation of the balloon in stage 3 , i.e. stage of ventricular tachycardia and raised blood pressure, usually causes the acute brain swelling, in which cerebral vasomotor paralysis (Langfitt, 1965) seems to play an important role. Evacuation in other stage is not followed by the brain swelling.

The present study was attempted to find correlation between ultrasonic attenuation and cerebral compression and acute brain swelling. Small balloon was placed in the parietal epidural space of the dog. Barium titanate ultrasonic transducer with a face diameter of $10 \mathrm{~mm}$ was fixed on the contralateral parietal region to receive the reflected beams from the temporal bone on the compressed side. Ultrasonic attenuation was measured during expansion and after evacuation of the epidural balloon. Respiration, ECG, cisternal pressure, venous pressure in the confluence, and venous flow from the confluence were recorded continuously.

Ultrasonic attenuation usually increased in stage 1, although decreased in a few cases, and always increased in stage 2. Following rapid evacuation of the balloon in stage 2 ultrasonic attenuation decreased promptly and reached to almost original level within several minutes, then decreased below the resting level in some cases. However, it always returned to the resting value several hours after evacuation. No brain swelling was recognized in these cases. If the balloon was deflated in stage 3 , ultrasonic attenuation decreased only temporarily. Several to 20 minutes after deflation it started to increase again in association with rapid rise in cisternal pressure and blood pressure and with prompt decrease in venous pressure and venous flow. This phenomenon is due to the acute brain swelling following rapid decompression. Attenuation remained at high value. Animals died usually 20 to 60 minutes after evacuation. Marked brain swelling and herniation were confirmed by autopsy.

However, evacuation in stage 3 did not always result in death of the animal. Following rebound increase on evacuation in stage 3 ultrasonic attenuation gradually decreased and returned to the resting level within several hours in some cases. These animals survived.

Expansion of an epidural balloon deforms intracranial contents and reduces cerebrospinal fluid space and vascular bed. These factors may explain the increase in ultrasonic attenuation during brain compression. Since grade of deformity and reduction of compressible space are different in each part of the brain, changes in u'trasonic attenuation during brain compression may be modified by several factors, such as ultrasonic wave length, direction and divergence of ultrasonic beam, and method of measurement of attenuation.

In order to analyze the significance of changes in ultrasonic attenuation in the acute brain swelling, precise morphological changes of the intracranial contents following evacuation must be studied. 